\title{
Effects of stimulus intensity on approach-avoidance conflict behavior'
}

JOHN W. DONAHOE AND VINCENT G. SCHULTE

UNIVERSITY OF KENTUCKY

Using FR approach and conflict chains and a light whose intensity increased or decreased within the FR as a function of responding, the present experiment found stimulus intensity to affect performance only when on-trial stimuli were discriminated from off-trial stimuli by permitting nonreinforced barpressing during the intertrial interval. These findings suggest a stimulus intensity, rather than a discriminative, origin of observed inequalities in the slopes of approach and avoidance gradients.

The purpose of this experiment was to evaluate the effects of the intensity of external stimulation on performance within approach and approach-avoidance conflict chains. In the runway situation, in which conflict behavior is typically studied, the experiment is conducted in a darkened room with a light located above the goal (Brown, 1948) or within the goal (Miller \& Kraeling, 1952). Thus, the latter portions of the runway are more brightly illuminated than the earlier portions. As a search of the literature has uncovered no studies in which the brightness relations were reversed, the possibility exists that the postulated differences in the shapes of the approach and avoidance gradients (Miller, 1959) may in part be functions of the particular brightness relations in the experiment, especially since appreciable stimulus intensity effects have been shown to occur in the runway (Brown, 1942).

In the present experiment, a runway situation was simulated by means of a fixed-ratio (FR) schedule. For half the Ss, the intensity of a light over the response bar increased as a function of responding; for the other half, the intensity decreased as a function of responding. In order to test the Perkins-Logan hypothesis (Logan, 1954; Perkins, 1953) regarding the origin of stimulus intensity effects, the bar was retracted between trials for half of the Ss (nondiscrimination condition - N) while the bar remained in the chamber between trials and intertrial responses were nonreinforced for the other half of the Ss (discrimination condition - D). According to the Perkins-Logan hypothesis, dynamism effects are attributed to generalization of inhibition from stimuli present during nonreinforcement to "on-trial" stimuli and, hence, stimulus intensity should be a variable only within Condition D.

Method

Ss were 32 male, 100 day old albino rats maintained at $80 \%$ of free feeding weight throughout the experiment.

The apparatus consisted of four identically constructed operant chambers, each chamber having a retractable bar, a pilot lamp $3 \mathrm{in}$, over the bar, and a foodcup. The operant chambers were enclosed within sound attenuatIng hulls with blowers to provide ventilation and an 85 dB white noise masking stimulus. Experimental events were controlled and responses were monitored by means of electromechanical switching and timing circuits located in an adjacent room.

Following adaptation to the laboratory and institution of the deprivation schedule, Ss were given two days of feeder training and two days of continuous reinforcement (60 45-mg pellets per day) under the same stimulus intensity which subsequently prevalled during reinforcement on FR trials. All Ss were then given 20 FR-20 trials per day for 18 days. For half the Ss, the light intensity changed in 10 equal $\log$ steps in an ascending (ASC) series from 3 to $20 \mathrm{ft}$-c every two responses. A descending (DESC) series was used for the other Ss. For half the ASC and DESC groups, the bar was retracted for $60 \mathrm{sec}$ between trials. For the other half of the ASC and DESC groups, responding during the intertrial interval postponed the onset of the next FR trial for $t s e c$. The value of $t$ was gradually increased to maintain an intertrial interval of approximately $60 \mathrm{sec}$ throughout training. The terminal value of $t$ was $50 \mathrm{sec}$. After the attainment of stable approach behavior, all Ss were given seven days of conflict training in which a $.5 \mathrm{sec}$ $.2 \mathrm{~mA}$ shock in addition to a food pellet was produced by the terminal response to FR-20.

Performance was measured by speed scores determined by computing the reciprocals of the first response latency and of the times required to complete every two responses thereafter. Thus, there were 11 measures within the FR trial-starting speed (SS) and 10 speeds for successive fractions of two responses.

Results and Discussion

Figure 1 shows the mean approach (AP), conflict (CF), and avoidance (AV) gradients during the last three days of training for Condition N. AV was obtained by subtracting CF from AP under the assumption that the gradients are independent (Miller, 1959). All gradients were increasing functions of proximity to reinforcement and their shapes do not appear to differ as a function of the ASC and DESC stimulus series. Analysis of variance confirmed that gradient shape was unchanged by stimulus intensity for all three gradients ( $p>.25$ in each case). although the AP and AV gradients differed in slope (F = 2.50 , df $=9 / 1008, p<.01) .2$ Thus, with a nondiscrimination procedure, external stimulus intensity had no measurable effect upon performance.

Figure 2 shows the AP, CF, and AV gradients during the last three days of training for Condition $D$. The di- 
rection of Intensity clearly affected the shape of the gradients when the "on-trial" stimuli were discriminated from the "off-trial" stimuli. Both the AP and AV gradients attained their maximas in the region of highest stimulus intensity independent of the proximity of reinforcement. The interaction of ratio fractions with direction of intensity change was significant for $A P(F=2.08$, $\mathrm{df}=9 / 189, \mathrm{p}<.05)$ and for $\mathrm{AV}(\mathrm{F}=2.27, \mathrm{df}=9 / 189, \mathrm{p}<$ $.05)$. Note that the AV gradient has a positive slope in the ASC group, but a predominantly negative slope in the DESC group.

The results of this study indicate that external stimulus intensity affects performance within a behavior chain only with a discrimination procedure and are, thereby, consistent with the Perkins-Logan analysis of dynamism. The nature of the stimulus intensity effect is such as to profoundly alter the shapes of the AP and AV gradients. To the extent that these findings apply to runway behavior, they suggest that implicit discrimination training is administered in the runway and, moreover, that existing theoretical accounts of conflict behavior may be subject to an alternative interpretation. That is, appetitive and aversive reinforcement may interact with stimulus intensity per se, and not necessarily with the discriminative or cue properties of the stimulus.

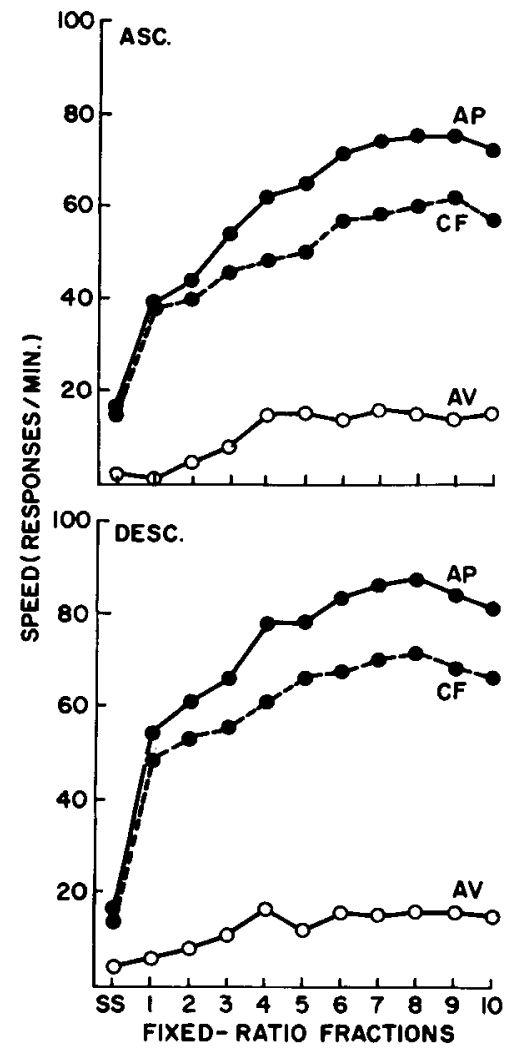

Fig. 1. Approach (AP), conflict (CF), and avoidance (AV) gradients obtained with a nondiscrimination procedure.

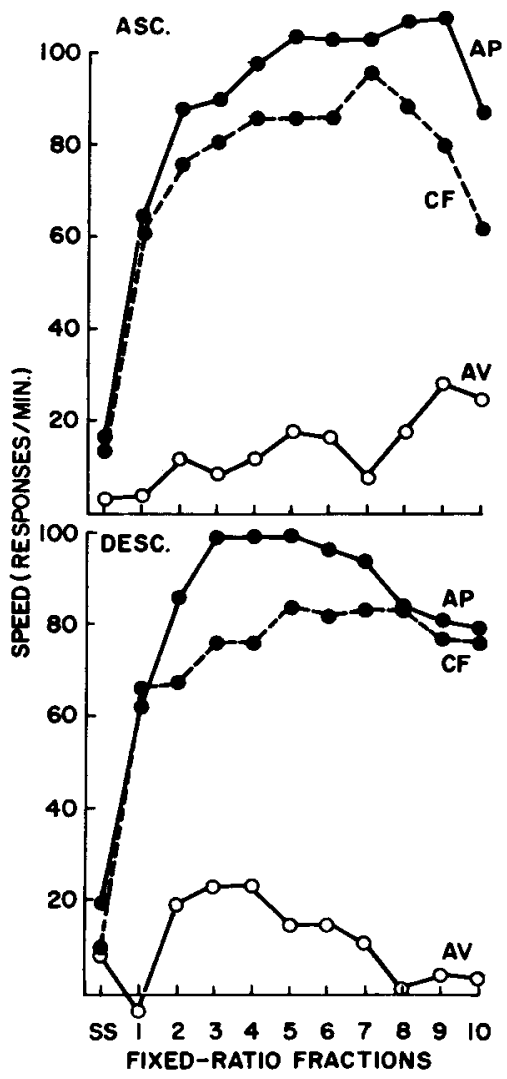

Fig. 2. Approach (AP), conflict (CF), and avoidance (AV) gradients obtained with a discrimination procedure.

\section{References}

Brown, J. S. The generalization of approach responses as a function of stimulus intensity and strength of motivation. J. comp. Psychol., 1942, 33, 209-226.

Brown, J. S. Gradients of approach and avoidance responses and their relation to level of motivation. J. comp. physiol. Psychol., $1948,41,450-465$.

Logan, F. A. A note on stimulus intensity dynamism (V). Psychol. Rev., 1954, 61, 77-80.

Miller, N. E. Liberalization of basic S-R concepts: Extensions to conflict behavior, motivation, and social learning. In $\mathrm{S}$. Koch (Ed.), Psychology: A study of a science, vol. 2. New York: McGraw-Hill, 1959.

Miller, N. E., \& Kraeling, D. Displacement: greater generalization of approach than avoidance in a generalized approach-avoidance conflict. J. exp. Psychol., 1952, 43, 217-221.

Perkins, C. C., Jr. The relation between conditioned stimulus intensity and response strength. J. exp. Psychol., 1953, 46, 225-231.

\section{Notes}

1. The research was supported by grant MH 10706 from the United States Public Health Service to the senior author. Acknowledgment is made to the Computing Center, University of Kentucky for data analysis and to Charles $\mathrm{A}$. Stewart for experimental assistance. 2. The findings reported here are a subset of a larger experiment concerned with additional variables and all significance tests make use of estimates of error variance derived from the entire experiment. 